\title{
Commentary on Seasonal Influenza Vaccine Dose Distribution in 195 countries (2004-2013): Little Progress in Estimated Global Vaccination Coverage
}

\author{
Bram Palache ${ }^{1,2^{*}}$ \\ ${ }^{1}$ FluPal Consultancy B.V., Bourgondischelaan 33, 1181DC Amstelveen, The Netherlands \\ ${ }^{2}$ International Federation of Pharmaceutical Manufacturers \& Associations, Chemin des Mines 9, P.O.Box 195, 1211 Geneva 20, Switzerland
}

*Corresponding author: Bram Palache, International Federation of Pharmaceutical Manufacturers \& Associations, Chemin des Mines 9 , P.O.Box 195, 1211 Geneva 20, Switzerland, E-mail: Bram.palache@ziggo.nl

Received date: January 29, 2016; Accepted date: February 16, 2015; Published date: February 19, 2015

Copyright: $\odot 2016$ Palache B, This is an open-access article distributed under the terms of the Creative Commons Attribution License, which permits unrestricted use, distribution, and reproduction in any medium, provided the original author and source are credited.

\section{Commentary}

The global influenza vaccine distribution data presented in this paper show a serious global underutilization of influenza vaccines. The influenza vaccine uptake figures show a huge gap between different regions of the world. Globally, more than $50 \%$ of influenza vaccines are distributed in the Americas compared to only 4\% in other regions, where more than half of the world's population live.

Although vaccine distribution is only a crude measure of vaccine uptake, the findings from this study agree with those of a Europeanwide survey of the influenza vaccination Venice II project [1,2], which show that the uptake rate for at-risk populations is still well below the WHO-recommended target of having $75 \%$ covered.

This trend is alarming given the potential of influenza vaccines to substantially reduce the annual disease burden and given their proven safety [2-4]. The underuse of influenza vaccines is a serious public health concern that needs to be addressed.

Research has consistently identified a variety of drivers and barriers to influenza vaccine uptake. Yet, many countries fail to address these factors. Even worse, such inaction contributes to the steady drop in the vaccine distribution rates that we have been witnessing in recent years, in some countries. As a consequence, there could be a potential impact related to vulnerable patients, including those suffering from chronic diseases, remaining unprotected against health complications associated with influenza.

Influenza virus, seasonal epidemiological variability and associated methodological complexities have resulted in variations of outcomes of clinical influenza vaccine effectiveness studies. For instance, based on a meta-analysis, some authors imply low vaccine effectiveness for the elderly [5], whereas others, in a re-analysis of the same data [6] found significant vaccine effectiveness against influenza-related complications (average $\sim 30 \%$ effectiveness with large dispersion), typical influenza-like illness ( $\sim 40 \%$ effectiveness), disease with confirmed virus infection ( $\sim 50 \%$ effectiveness), and biological vaccine efficacy against infection $(\sim 60 \%)$.

Although the large variability in study outcomes may offer room for scientific debate about the "true" vaccine effectiveness in any particular year and geographical area, overall, the benefits of influenza vaccination are well documented $[2,3]$ and form the scientific basis for current policy recommendations. Because overall, the influenza vaccines in the USA and Europe are the same, vaccine effectiveness cannot explain the disparity in trends of vaccine distribution rates in the 2 respective continents.
One may doubt whether new influenza vaccines with an improved effectiveness profile will indeed lead to better uptake. Interestingly, Preaud et al. [2] compared the health benefits between the current vaccines with an ideal $75 \%$ coverage rate and hypothetical vaccines of higher vaccine effectiveness $(80 \%)$ with current vaccination rates. Overall, the gained health benefits of both scenarios were gloomily similar.

The findings regarding poor influenza prevention are in strong contrast with global health initiatives, which recognize the need to keep people healthy throughout their lives. In addition, the drop in uptake rates for influenza vaccines undermines the global efforts and investments made by WHO's global surveillance networks GISRS [7], Global Action Plan for Influenza [8] and the Pandemic Influenza Preparedness programs [9]. These initiatives ensure that the influenza viruses selected for inclusion in seasonal influenza vaccines are updated based on which viruses are circulating, how they are spreading, and how well the new vaccines components protect against newly identified viruses, while monitoring any pandemic that may occur. Finally, seasonal influenza vaccination infrastructure can also be considered as a measure for pandemic preparedness.

Decision makers should continue to improve their understanding of the overwhelming evidence that influenza vaccines are safe and effective. Today, the scientific evidence available on best practices for influenza vaccination does not seem to guide countries' responses to annual influenza outbreaks. On the contrary, political will and health policy change seem to be stronger determining factors.

Given the current state of poor influenza prevention globally, there is an urgent need to reconsider the national efforts and policies. Firstly, political will and commitment are absolutely imperative for influenza prevention to be realized. Likewise, healthcare professionals should take note of the statistics in this report and use them in developing their plans to increase influenza vaccination rates in the future. Secondly, spreading the word on the benefits of influenza vaccines is important. There are many tactics to reach the right audience, such as social media, targeting specific atrisk populations rather than the population at large. Social media may offer opportunities to address potential behavior attitudes through a peer-to-peer shareable social media driven campaign.

To conclude, improved prevention of influenza is a societal, medical and ethical need, which can already be achieved by efficiently implementing existing policy recommendations. However to achieve this, different stakeholders, such as national policymakers, governments, health professionals and patient groups should work in partnership to ensure that at-risk groups will be better protected against this preventable infectious disease and its complications. 
Citation: Palache B (2016) Commentary on Seasonal Influenza Vaccine Dose Distribution in 195 countries (2004-2013): Little Progress in Estimated Global Vaccination Coverage. J Vaccines Vaccin 7: 308. doi:10.4172/2157-7560.1000308

Page 2 of 2

Providing and taking the seasonal influenza vaccine is therefore everyone's responsibility.

\section{References}

1. Mereckiene J, Cotter S, O’Flanagan D (2014) National seasonal influenza vaccination survey for 2012- 13 influenza season in EU/EEA (provisional data) In: VENICE III Consortium.

2. Preaud E, Durand L, Macabeo B, Farkas N, Sloesen B, et al. (2014) The Vaccines Europe influenza working group. Annual public health and economic benefits of seasonal influenza vaccination: a European estimate. BMC Public Health, 14: 813.

3. Kostova D, Reed C, Finelli L, Cheng PY, Gargiullo PM (2013) Influenza Illness and Hospitalizations Averted by Influenza Vaccination in the United States, 2005-2011, Plos one.
4. WHO (2012) Vaccines against influenza WHO position paper. Weekly epidemiological record No. 47, 87: 461-476.

5. Jefferson T, Di Pietrantonj C, Al-Ansary LA, Ferroni E, Thorning S, et al. (2010) Vaccines for preventing influenza in the elderly. Cochrane Database Syst Rev, 17: 2.

6. Beyer WEP, McElhaney J, Smith DJ, Monto AS, Nguyen-Van-Tam JS, et al. (2013) Cochrane re-arranged: Support for policies to vaccinate elderly people against influenza. Vaccine, 31: 6030-6033

7. http://www.who.int/csr/disease/OP_GISRS_FINAL.pdf.

8. http://www.who.int/influenza_vaccines_plan/en/.

9. http://www.who.int/influenza/resources/pip_framework/en/. 